Tema: Aciaria elétrica

\title{
ESTUDO DA ADIÇÃO DE POEIRA DE ACIARIA ELÉTRICA EM FERRO-GUSA LÍQUIDO*
}

\author{
Vicente de Paulo Ferreira Marques Sobrinho ${ }^{1}$ \\ Estéfano Aparecido Vieira ${ }^{1}$ \\ José Roberto de Oliveira ${ }^{1}$ \\ Victor Bridi Telles ${ }^{2}$ \\ Felipe Fardin Grillo ${ }^{2}$ \\ Jorge Alberto Soares Tenório ${ }^{3}$ \\ Denise Crocce Romano Espinosa ${ }^{4}$
}

\section{Resumo}

Esta pesquisa tem como objetivo estudar a incorporação da massa da poeira de aciaria elétrica (PAE), mediante adição em ferro-gusa $(1,78 \% \mathrm{Si})$ à temperatura de 1.400 graus Celsius. A PAE é proveniente de uma usina siderúrgica produtora de aços longos. A adição da PAE ao ferro-gusa líquido será sob a forma como recebido, sob a forma de briquetes sem agitação do ferro-gusa líquido e sob a forma de briquetes com agitação do ferro-gusa líquido. Previamente, a PAE foi caracterizada utilizando-se as seguintes técnicas: análise química, análise granulométrica, área de superfície específica, difração de raios- $X$, microscopia eletrônica de varredura (MEV) e análise de micro-regiões por EDS. A realização dos experimentos de fusão, em escala de laboratório, ocorreu em um forno vertical tubular com controlador de temperatura. Os experimentos de fusão para avaliar a incorporação da massa de PAE utilizaram cadinhos de grafite. Após o resfriamento, ferro gusa e escória remanescentes no cadinho foram pesados objetivando efetuar um balanço de massa. Um fluxo de gás inerte (argônio) foi mantido no interior do forno durante a realização dos experimentos.

Palavras-chave: Resíduos siderúrgicos; Poeiras; Reciclagem.

\section{STUDY OF THE ADDITION OF ELECTRIC ARC FURNACE DUST IN HOT METAL Abstract}

This research aims to study the incorporation of the mass of electric arc furnace dust (EAFD), by addition in hot metal $(1,78 \% \mathrm{Si})$ at a temperature of 1,400 degrees Celsius. The EAFD is from a steel plant producing long steel. The addition of the EAFD was as received, in the form of briquettes without agitation of the hot metal and in the form of briquettes with agitation of the hot metal. Previously, the EAFD was characterized using the following techniques: chemical analysis, size analysis, X-ray diffraction, scanning electron microscopy (SEM) and energy dispersive spectroscopy (EDS) microanalysis. The achievement of fusion experiments in laboratory scale, took place in a vertical tubular furnace with temperature control. The fusion experiments to assess the incorporation of EAFD mass used graphite crucibles. After cooling, the hot metal and the slag, remaining in the crucible, were weighed to do a mass balance. A flow of inert gas (argon) was maintained inside the furnace during the experiments.

Keywords: Steelmaking residue; Dust; Recycling.

1 Engenheiro metalurgista, Professor, IFES, Vitória, ES, Brasil.

2 Doutorando em Engenharia Metalúrgica e de Materiais, Escola Politécnica, USP, São Paulo, SP, Brasil.

3 Engenheiro metalurgista, Professor Titular, Escola Politécnica, Universidade de São Paulo, SP, Brasil.

4 Engenheiro metalurgista, Professora, Escola Politécnica, Universidade de São Paulo, SP, Brasil.

* Contribuição técnica ao $45^{\circ}$ Seminário de Aciaria - Internacional, 25 a 28 de maio de 2014, Porto Alegre, RS, Brasil. 


\section{INTRODUÇÃO}

O setor siderúrgico gera uma diversidade de resíduos sólidos, efluentes líquidos e emissões gasosas, nas diversas etapas de seu processamento. A poeira de aciaria elétrica (PAE), gerada no forno elétrico a arco, representa um dos maiores problemas pelo seu conteúdo em elementos químicos, tais como zinco, ferro, cromo, cádmio, entre outros, emitidos à atmosfera durante a fabricação do aço [1].

Independente do tipo de forno ou do processo utilizado, fabricar aço é uma operação que impacta o meio ambiente em função de emissão de uma grande quantidade de poeira [1]. Vários autores [2-7] têm relatado os índices da quantidade gerada de poeira de aciaria com relação à produção de aço. Tais valores mostram que entre 15 $\mathrm{kg}$ a $20 \mathrm{~kg}$ de PAE são gerados para cada tonelada de aço que é produzida nos fornos elétricos a arco.

No mundo, a quantidade de aço produzida via forno elétrico a arco vem aumentando a cada ano, consequentemente, a quantidade gerada de PAE também está aumentando [8].

A poeira de aciaria elétrica é classificada como resíduo perigoso no Brasil de acordo com os critérios descritos na NBR 10.004 [9], bem como em outros países produtores de aço, como Estados Unidos [10,11], Japão [12] e União Europeia [13]. Devido a esta classificação, a PAE não pode ser depositada em aterros não controlados, de tal forma que toneladas de PAE encontram-se provisoriamente armazenadas em aterros industriais aguardando por uma devida solução.

Diante do exposto, é válido afirmar que com a crescente quantidade de geração de PAE, tornar-se oportuno e necessário um estudo de alternativas de reciclagem da PAE, tal que o seu reaproveitamento desde que em bases econômicas aceitáveis, reduza custos quando do uso interno ou gere receitas quando o uso for externo [14].

\section{MATERIAIS E MÉTODOS}

Para a execução deste projeto, foram desenvolvidas as seguintes etapas:

- aquisição dos resíduos e matérias-primas;

- caracterização física e química da PAE;

- realização dos testes de adição da PAE no estado "como recebido", adição de PAE sob a forma de briquetes sem agitação do banho e adição de PAE sob a forma de briquetes com agitação do ferro-gusa líquido; e

- após o resfriamento do forno à temperatura ambiente, será feita a pesagem do ferro-gusa e da escória para que seja feito um balanço de massa para avaliar a incorporação de massa no ferro gusa.

Foram utilizadas as seguintes matérias-primas para a realização dos ensaios:

- poeira de aciaria elétrica; e

- ferro-gusa.

Foram coletados $100 \mathrm{~kg}$ de PAE, $100 \mathrm{~kg}$ de ferro-gusa em indústrias da grande Vitória, no estado do Espírito Santo a saber:

- poeira de aciaria elétrica: ArcellorMittal/Cariacica; e

- ferro gusa: Fundição Ucrânia localizada no município da Serra.

De posse das matérias-primas, a etapa seguinte foi a caracterização química e física das matérias-primas principais, mediante várias técnicas de caracterização, sendo elas:

- análise química;

- análise granulométrica;

* Contribuição técnica ao $45^{\circ}$ Seminário de Aciaria - Internacional, 25 a 28 de maio de 2014, 


\section{ACJARIA}

- área de superfície específica;

- caracterização da forma das partículas da poeira mediante o uso de microscopia ótica e eletrônica de varredura;

- análise por dispersão de energia; e

- difração de raios-X.

A análise química fez-se necessária para se confirmar os teores dos vários elementos presentes nos resíduos.

$\mathrm{Na}$ análise granulométrica das amostras de resíduo foi empregado um analisador de tamanho de partículas (Malvern 2600-LC), que se baseia no espalhamento de luz causado pelas partículas quando incide sobre as mesmas um feixe de raio laser.

A difração de raios- $X$ foi utilizada para a determinação dos principais compostos presentes nas amostras de PAE.

As microscopias óptica e eletrônica de varredura foram utilizadas para analisar a morfologia e a estrutura interna das partículas da PAE. Inicialmente foram feitas imagens no MEV somente do exterior das partículas da PAE.

Para fazer uma análise qualitativa e semi-quantitativa dos elementos presentes na PAE, foi realizada uma análise por dispersão de energia (EDS), utilizando um detector acoplado ao MEV.

Para os experimentos de estudo da injeção de PAE, o ferro gusa com a composição conhecida (1,78\% de silício) na quantidade de 802,60 a 820,00g, foi carregado em um cadinho de alumina. Este cadinho será então levado até um forno vertical tubular. Em seguida foi injetado argônio na câmara de aquecimento, para evitar a oxidação do ferro-gusa.

Quando a temperatura do banho atingiu a temperatura de trabalho $\left(1.400^{\circ} \mathrm{C}\right)$, a PAE foi adicionada. A temperatura é medida e controlada pelo sistema de medição e controle do próprio forno. Foram retiradas amostras do metal e da escória quinze minutos após a adição da mistura através de um amostrador a vácuo e em seguida o forno foi desligado. Após resfriamento, metal e escória remanescentes no cadinho foram pesados objetivando efetuar um balanço de massa. De posse dos resultados, os mesmos foram comparados, para se medir a eficiência do processo.

\section{RESULTADOS E DISCUSSÃO}

O resultado da análise química da PAE é apresentado na Tabela 1.

Tabela 1. Composição química da PAE

\begin{tabular}{|c|c|c|c|}
\hline Elemento Químico & Composição (\%) & Elemento Químico & Composição (\%) \\
\hline $\mathrm{Fe}_{\text {total }}$ & 38,9 & $\mathrm{~S}$ & 0,88 \\
\hline $\mathrm{Zn}_{\text {total }}$ & 11,7 & $\mathrm{Al}$ & 0,32 \\
\hline $\mathrm{Ca}$ & 3,06 & $\mathrm{P}$ & 0,14 \\
\hline $\mathrm{Si}$ & 1,66 & $\mathrm{Cu}$ & 0,28 \\
\hline $\mathrm{Mn}$ & 2,27 & $\mathrm{Cr}$ & 0,21 \\
\hline $\mathrm{Mg}$ & 1,33 & $\mathrm{Ti}$ & 0,08 \\
\hline $\mathrm{Cl}$ & 4,64 & $\mathrm{Br}$ & 0,13 \\
\hline $\mathrm{K}$ & 1,87 & $\mathrm{Sn}$ & 0,07 \\
\hline $\mathrm{Pb}$ & 1,48 & $\mathrm{Cd}$ & 0,03 \\
\hline
\end{tabular}

$\mathrm{Fe}_{\text {total }}$ corresponde ao metal na forma de Fé, $\mathrm{Fe}_{3} \mathrm{O}_{4}, \mathrm{Fe}_{2} \mathrm{O}_{3}$ e $\mathrm{FeO} ; \mathrm{Zn}$ total corresponde ao metal na forma de $\mathrm{Zn}$, $\mathrm{ZnO}$ e de zinco associado com o Fe $\left(\mathrm{ZnFe}_{2} \mathrm{O}_{4}\right)$

* Contribuição técnica ao $45^{\circ}$ Seminário de Aciaria - Internacional, 25 a 28 de maio de 2014, 
O teor de Fe e o teor de Zn da PAE são compatíveis com os teores de ferro $(24,9$ a $46,9 \%)$ e com os teores de zinco (11,12 a 26,9\%) apresentados pelas siderúrgicas americanas [15].

O resultado da análise do tamanho das partículas está representado na Figura 1.

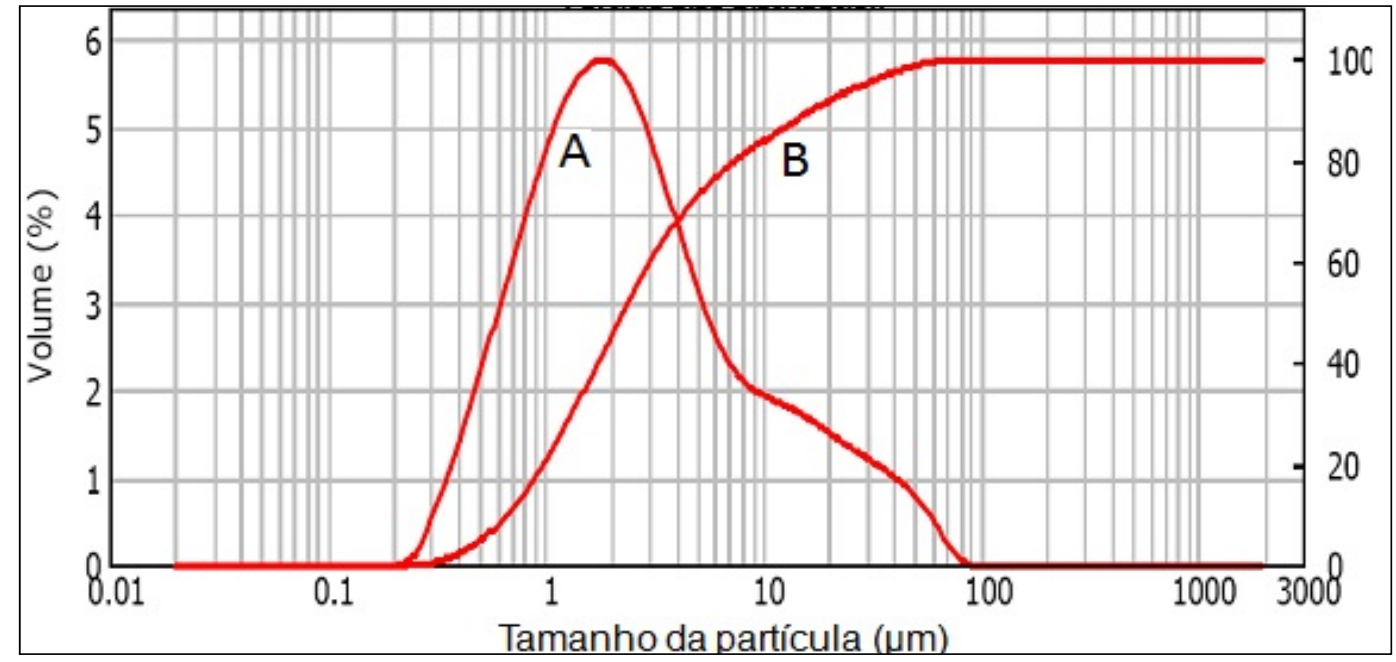

Figura 1. Distribuição do tamanho das partículas.

A linha (A) representa o percentual de distribuição do tamanho das partículas, a linha (B) representa o percentual acumulado.

No relatório do ensaio emitido pelo equipamento, observa-se que $10 \%$ da amostra possui diâmetro da partícula menor que $0,672 \mu \mathrm{m}$, e $90 \%$ do material apresenta granulometria inferior a 17,070 $\mu \mathrm{m}$. O resultado da área superficial específica das partículas foi de $3,81 \mathrm{~m}^{2} / \mathrm{g}$ e o diâmetro médio da partícula 2,281 $\mu \mathrm{m}$.

A Figura 2 apresenta o espectro de difração de raios-X da PAE.

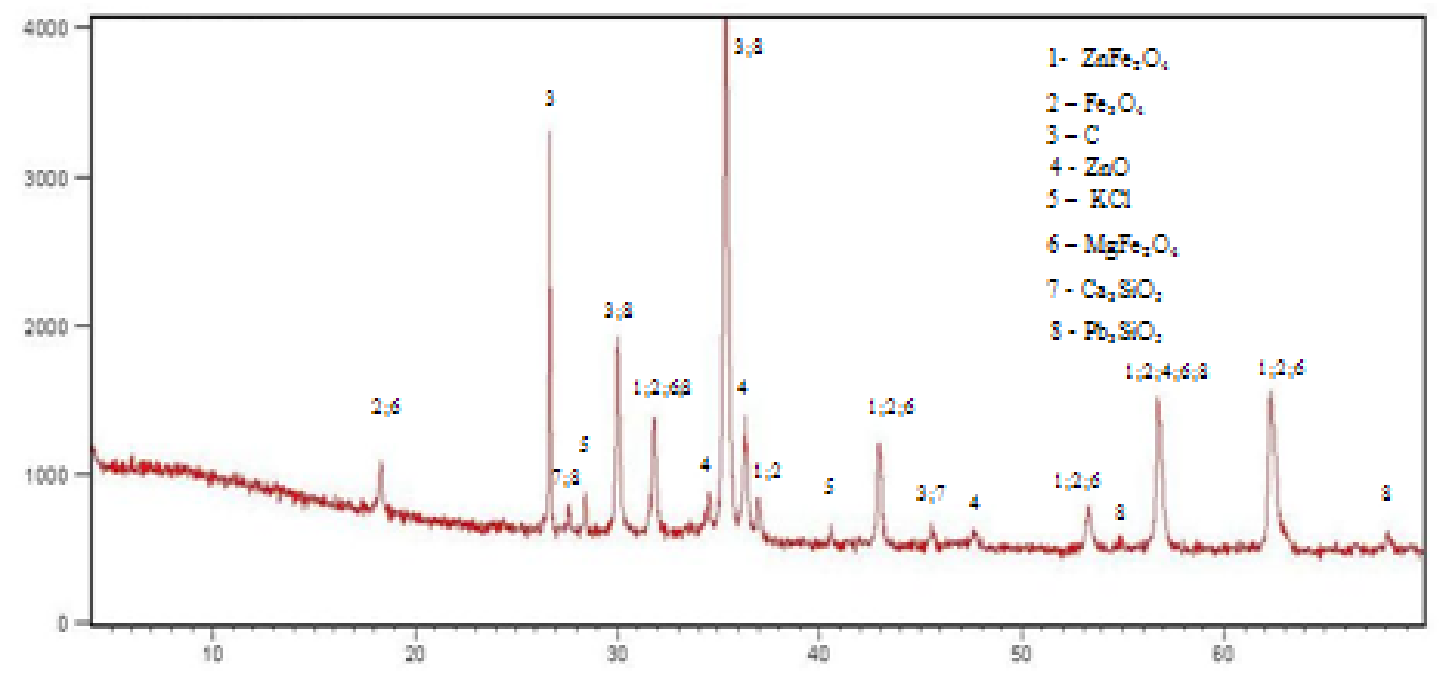

Figura 2. Espectro de difração de raios-X da PAE.

Observa-se na figura acima a predominância da Magnetita $\left(\mathrm{Fe}_{3} \mathrm{O}_{4}\right)$, Magnesioferrita $\left(\mathrm{MgFe}_{2} \mathrm{O} 4\right)$ e Franklinita $\left(\mathrm{ZnFe}_{2} \mathrm{O}_{4}\right)$.

A Figura 3 apresenta micrografias apresentando a morfologia da PAE.

* Contribuição técnica ao 45 Seminário de Aciaria - Internacional, 25 a 28 de maio de 2014, 

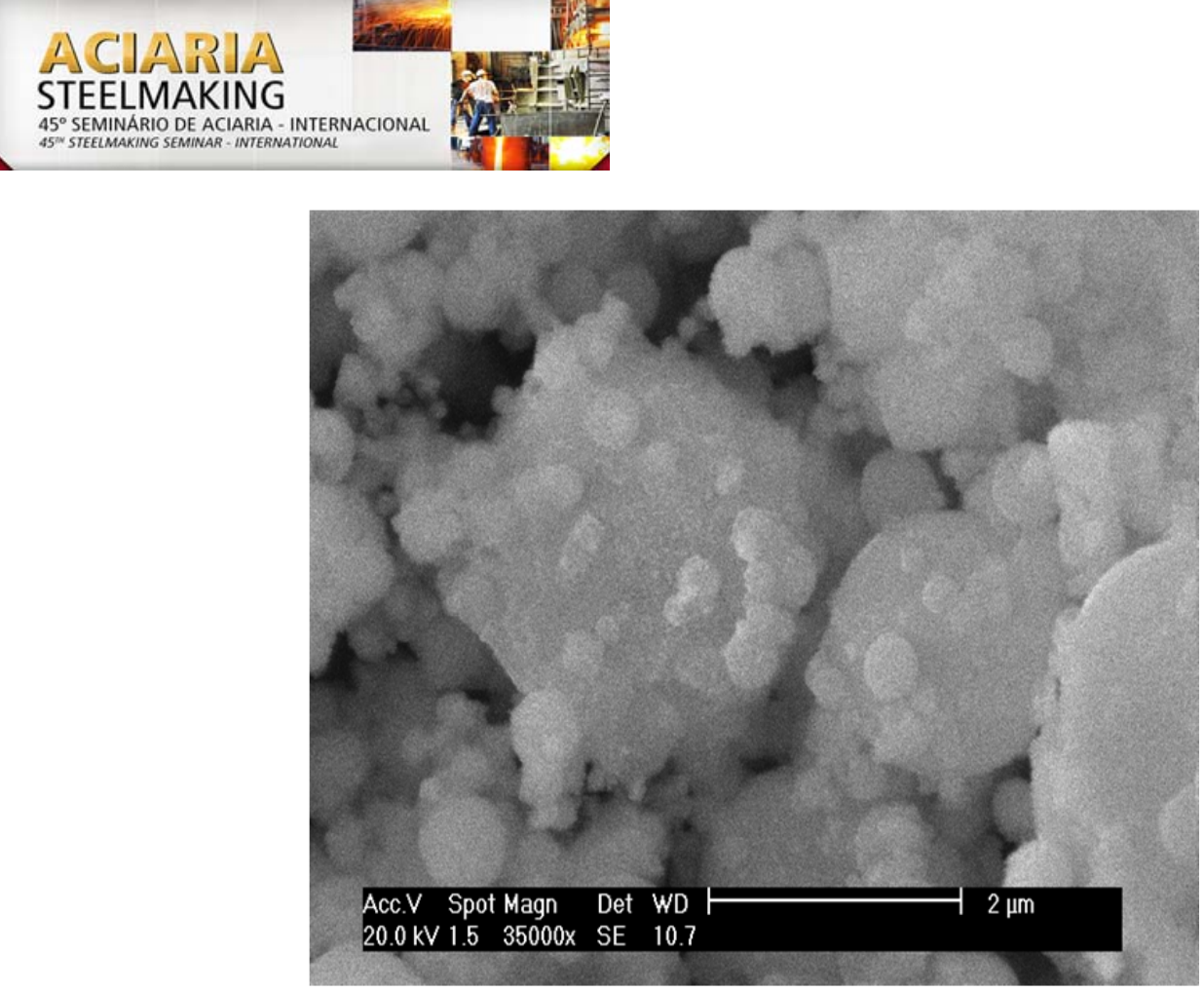

Figura 3. Microscopia Eletrônica da PAE.

Verifica-se que a PAE é constituída predominantemente de partículas de formato esférico, formato este que está diretamente relacionado com o mecanismo de formação das poeiras.

A Tabela 2 apresenta os resultados dos experimentos efetuados com a adição de $\mathrm{PAE}$ em ferro-gusa líquido à temperatura de $1.400^{\circ} \mathrm{C}$ :

Tabela 2. Adição de $P A E$ em ferro-gusa líquido à temperatura de $1.400^{\circ} \mathrm{C}$

\begin{tabular}{|c|c|c|c|}
\hline & $\begin{array}{c}\text { Adição de PAE } \\
\text { como recebido }\end{array}$ & $\begin{array}{c}\text { Adição de PAE } \\
\text { sob a forma de } \\
\text { briquete }\end{array}$ & $\begin{array}{c}\text { Adição de PAE sob } \\
\text { a forma de briquete } \\
\text { com agitação do } \\
\text { banho }\end{array}$ \\
\hline Massa inicial do gusa (g) & 818,60 & 820,00 & 802,60 \\
\hline Massa do briquete (g) & 96,60 & 96,80 & 94,76 \\
\hline Massa final do gusa (g) & 832,74 & 826,37 & 816,00 \\
\hline Massa da escória (g) & 40,10 & 45,40 & 37,40 \\
\hline \% de escória & 4,82 & 5,49 & 4,58 \\
\hline Massa da PAE volatilizada (g) & 42.36 & 45,03 & 43,96 \\
\hline \% de PAE volatilizada & 43,85 & 46,52 & 46,39 \\
\hline Massa de gusa incorporado (g) & 14,14 & 6,37 & 13,40 \\
\hline \% gusa incorporado & 1,73 & 0,78 & 1,67 \\
\hline
\end{tabular}

Observa-se que o experimento com adição de PAE como recebido apresenta o melhor resultado de incorporação à massa do ferro-gusa final combinado com o menor percentual de massa de PAE volatilizada. O experimento de adição de PAE na forma de briquete com agitação apresenta o menor percentual de massa de escória.

O resultado da análise química semi-quantitativa da PAE volatilizada referente ao experimento de adição de PAE como recebido é apresentado na Tabela 3.

* Contribuição técnica ao 450 Seminário de Aciaria - Internacional, 25 a 28 de maio de 2014, 
Tabela 3. Composição química da PAE volatilizada

\begin{tabular}{|c|c|c|c|}
\hline Substâncias & $\begin{array}{c}\text { Composição } \\
(\%)\end{array}$ & $\begin{array}{c}\text { Substâncias } \\
\text { Composição } \\
(\%)\end{array}$ \\
\hline $\mathrm{Fe}_{\text {total }}$ & 1,25 & $\mathrm{~S}$ & 0,03 \\
\hline $\mathrm{Zn}_{\text {total }}$ & 64,30 & $\mathrm{Al}$ & 0,66 \\
\hline $\mathrm{Ca}$ & 0,19 & $\mathrm{P}$ & 0,04 \\
\hline $\mathrm{Si}$ & 0,97 & $\mathrm{Cu}$ & 0,22 \\
\hline $\mathrm{Mn}$ & 0,40 & $\mathrm{Cr}$ & 0,28 \\
\hline $\mathrm{Br}$ & 0,00 & $\mathrm{Ni}$ & 0,51 \\
\hline $\mathrm{Cl}$ & 12,97 & $\mathrm{Mg}$ & 0,08 \\
\hline $\mathrm{K}$ & 2,16 & $\mathrm{Sn}$ & 0,00 \\
\hline $\mathrm{Pb}$ & 5,32 & $\mathrm{Cd}$ & 0,28 \\
\hline
\end{tabular}

$\mathrm{Fe}_{\text {total }}$ Corresponde ao metal na forma de $\mathrm{Fe}, \mathrm{Fe}_{3} \mathrm{O}_{4}, \mathrm{Fe}_{2} \mathrm{O}_{3}$ e FeO; $\mathrm{Zn}$ total corresponde ao metal na forma de $\mathrm{Zn}$, $\mathrm{ZnO}$ e de zinco associado com o Fe $\left(\mathrm{ZnFe}_{2} \mathrm{O}_{4}\right)$.

Pode-se observar que o teor de Fe diminui (de 38,9\% para 1,25\%) e o teor de $\mathrm{Zn}$ aumenta (de $11,7 \%$ para 64,30\%) quando comparados com os valores apresentados pela PAE como recebido. O teor de zinco da PAE volatilizada $(64,30 \%)$ é superior ao teor de zinco do concentrado de minério importado (52\%) e ao teor dos minérios nacionais (12\% a $39 \%)$.

A Figura 4 apresenta o espectro de difração de raios-X da PAE volatilizada.

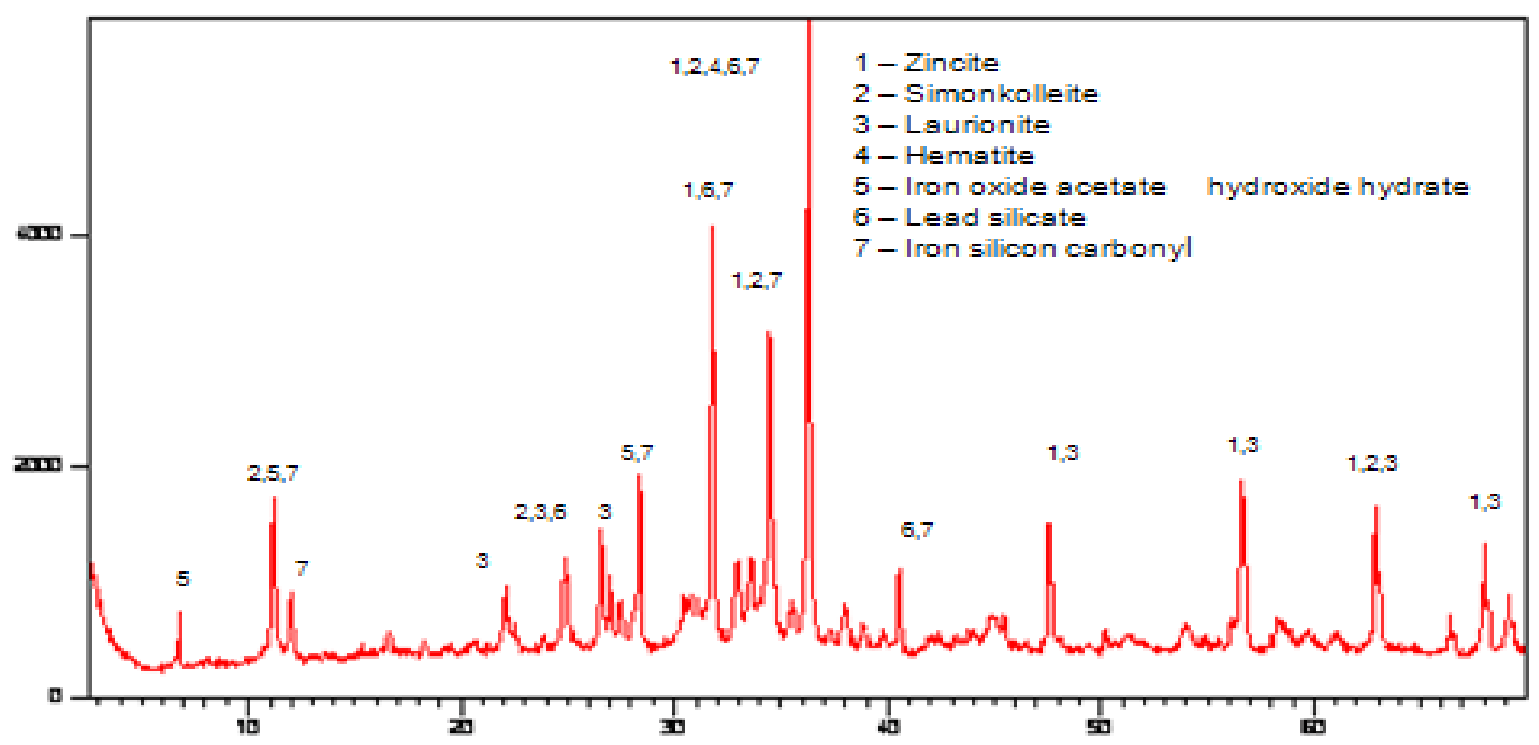

Figura 4. Espectro de difração de raios-X da PAE volatilizada.

Observa-se a predominância de óxido de zinco ( $Z n O)$, do composto denominado Simonkolleite $\left(\mathrm{Zn}_{5}(\mathrm{OH})_{8} \mathrm{Cl}_{2} \cdot \mathrm{H}_{2} \mathrm{O} / \mathrm{ZnCl}_{2} \cdot 4 \mathrm{Zn}(\mathrm{OH})_{2} \cdot \mathrm{H}_{2} \mathrm{O}\right)$ e do composto denominado Laurionite $(\mathrm{PbCl}(\mathrm{OH}))$. A presença de Magnetita $\left(\mathrm{Fe}_{3} \mathrm{O}_{4}\right)$, Magnesioferrita $\left(\mathrm{MgFe}_{2} \mathrm{O} 4\right)$ e Franklinita $\left(\mathrm{ZnFe}_{2} \mathrm{O}_{4}\right)$ predominantes na PAE como recebido, não foi detectada.

O resultado da análise química semi-quantitativa da escória referente ao experimento de adição de PAE como recebido é apresentado na Tabela 4.

* Contribuição técnica ao $45^{\circ}$ Seminário de Aciaria - Internacional, 25 a 28 de maio de 2014, 
Tabela 4. Composição química da escória

\begin{tabular}{|c|c|c|c|}
\hline Substâncias & Composição (\%) & Substâncias & Composição (\%) \\
\hline $\mathrm{Fe}$ & 10,52 & $\mathrm{~S}$ & 0,13 \\
\hline $\mathrm{Zn}$ & 4,58 & $\mathrm{Al}$ & 3,73 \\
\hline $\mathrm{Ca}$ & 4,70 & $\mathrm{P}$ & 0,05 \\
\hline $\mathrm{Si}$ & 29,09 & $\mathrm{Cl}$ & 0,49 \\
\hline $\mathrm{Mn}$ & 2,16 & $\mathrm{Cu}$ & 0,02 \\
\hline $\mathrm{Br}$ & 0,00 & $\mathrm{Ni}$ & 0,43 \\
\hline $\mathrm{Cl}$ & 0,49 & $\mathrm{Mg}$ & 4,04 \\
\hline $\mathrm{K}$ & 0,88 & $\mathrm{Mo}$ & 0,65 \\
\hline $\mathrm{Pb}$ & 1,02 & $\mathrm{Cd}$ & 0,31 \\
\hline
\end{tabular}

Observa-se na escória a presença de teores de Fe e Zn. A ocorrência de teores de $\mathrm{Pb}$ e $\mathrm{Cd}$ na escória, caracterizam a mesma como um resíduo sólido perigoso classe I.

\section{CONCLUSÃO}

A PAE estudada é constituída predominantemente de partículas de formato esférico, formato este que está diretamente relacionado com o mecanismo de formação das poeiras.

O experimento com adição de PAE como recebido apresenta o melhor resultado de incorporação à massa do ferro-gusa final combinado com o menor percentual de massa de PAE volatilizada. O experimento de adição de PAE na forma de briquete com agitação apresenta o menor percentual de massa de escória.

O teor de zinco da PAE volatilizada (64,30\%) é superior ao teor de zinco do concentrado de minério importado $(52 \%)$ e ao teor de zinco dos minérios nacionais $(12 \%$ a $39 \%)$.

A presença de $\mathrm{Pb}$ e $\mathrm{Cd}$ na escória, caracterizam a mesma como um resíduo sólido perigoso classe I.

\section{Agradecimentos}

Instituto Federal do Espírito Santo - IFES; Escola Politécnica da Universidade de São Paulo - Epusp; Fapesp; ArcellorMital Cariacica; Fundição Ucrânia e Harsco Metals.

\section{REFERÊNCIAS}

1 Silva MC. Reciclagem de pó de aciaria elétrica como matéria-prima na fabricação de materiais cerâmicos argilosos: controle das emissões atmosféricas de zinco [tese de doutorado]. Porto Alegre: Universidade Federal do Rio Grande do Sul; 2006. p.157.

2 Sobrinho PJN, Espinosa DCR, Tenório JAS. Caracterização da poeira gerada na fabricação de aço inoxidável visando a sua reciclagem. Metalurgia e Materiais. 2000;53(4):257-263.

3 Hagni AM, Hagni R De, Demars C. Mineralogical Characteisiticas of Eletric Arc Furnace Dusts. JOM.1991; April:28-30.

4 Nolasco Sobinho PJ, Espinosa DCR, Tenório JAS. Characterization of dusts and sludges generatede during stainless steel production in Brazilian industries. Iromaking and Steelmaking. 2003;3(1):11-17.

5 Takano C, Capocchi JDT, Nascimento RC, Mourão MB, Lenz G, Santos DM. A reciclagem de resíduos siderúrgicos sólidos. seminário nacional sobre reuso/reciclagem

* Contribuição técnica ao $45^{\circ}$ Seminário de Aciaria - Internacional, 25 a 28 de maio de 2014, 
de resíduos sólidos industriais. São Paulo: Secretaria do Meio Ambiente do Estado de São Paulo; 2000.

6 Huber JC, Patisson F, Rocabois P, Birat JP, Ablitzer D. Some means to reduce emissions and improve the recovery of electric arc furnace dust by controlling the formation mechanisms. In: REWAS; 1999. p.1483-92.

7 Dominguez EA, Ullmann R. "Ecological bricks" made with clays and steel dust pollutants. Applied Clay Science. 1996;11:237-249.

8 Mantovani MC. Caracterização de poeiras geradas em fornos elétricos a arco e seu estudo quando aglomeradas na forma de pelotas auto-redutoras [tese de doutorado]. São Paulo: Escola Politécnica, USP; 1998. p.165.

9 Associação Brasileira de Normas Técnicas. NBR 10.004: Resíduos Sólidos Classifcação. Segunda Edição. Rio de Janeiro; 2004.

$10 \mathrm{Li} \mathrm{CL}$, Tsai MS. Mechanism of spinel ferrite dust formation in electric arc furnace steelmaking. ISIJ International, 1993;33(2):284-290.

11 Ruiz O, Clemente C, Alonso M, Alguacil FJ. Recycling of na electric arc furnace flue dust to obtain high grade ZnO. Journal of Hazardous Material. 2007;141(1):33-36.

12 Zhang Y; Kasai Eiki, Nakamura Takashi. Vaporization behavior of zinc from the FeO-CaO- $\mathrm{SiO}_{2}-\mathrm{Al}_{2} \mathrm{O}_{3}$. ISIJ International. 2005;45(12):1813-19.

13 Pelino M, Karamanov A, Pisciella P, Crisucci S, Zonetti D. Vitrification of electric arc furnace dusts. Waste Management. 2002;22:945-949.

14 Marques Sobrinho VPF, Oliveira JR, Tenório JAS, Espinosa DCR. Reciclagem de poeira de aciaria a arco elétrico. In: $42^{\circ}$ Seminário de Aciaria da ABM; 2011; Salvador, Brasil. São Paulo: ABM; 2011. p. 609-618.

15 Nyrenda R. The processing of steelmaking flue-dust: a review. Minerals Engineering. 1991;4(7-11):1003-25.

* Contribuição técnica ao $45^{\circ}$ Seminário de Aciaria - Internacional, 25 a 28 de maio de 2014, 